\title{
纳米铁颗粒及其复合材料的界面设计及环境修复应用
}

\author{
苏莉，杨建平，兰悦，王连军，江 莞 \\ (东华大学 材料科学与工程学院, 纤维材料改性国家重点实验室, 上海 201620)
}

\begin{abstract}
摘 要: 近年来, 纳米铁颗粒 (纳米零价铁)因其优异的催化/还原性能, 并且价廉、环境友好, 已成为主要的环境修复 材料之一。目前, 纳米铁颗粒主要用于水体修复, 如: 重金属离子去除、有机物污染物降解和无机阴离子催化还原 等。纳米铁颗粒易团聚和结构单一等问题会导致其活性低、稳定性差和去除种类单一。为了克服上述问题, 迫切需 要研究纳米铁颗粒的界面设计。本文重点阐述纳米铁颗粒及其复合材料的可控制备、界面设计、在重金属去除和 硝酸根去除转化中的应用以及在环境修复中的未来发展方向。
\end{abstract}

关 键 词: 纳米铁颗粒; 界面设计; 吸附应用; 环境电催化; 环境修复; 综述

中图分类号: TQ174 文献标志码: A

\section{Interface Design of Iron Nanoparticles for Environmental Remediation}

\author{
SU Li, YANG Jianping, LAN Yue, WANG Lianjun, JIANG Wan
}

(State Key Laboratory for Modification of Chemical Fibers and Polymer Materials, College of Materials Science and Engineering, Donghua University, Shanghai 201620, China)

\begin{abstract}
Recently, iron nanoparticles have become one of the main research materials for environmental remediation due to the excellent catalytic/reduction performance, low cost, and environmental friendliness. At present, the research of iron nanoparticles mainly focuses on the heavy metal removal, organic pollution degradation, inorganic anion catalytic reduction in sewage system, etc. However, there still exist some problems, such as low removal efficiency, poor stability, and single removal on account of several self-defects of agglomeration and tedious structures. Hence, this review focuses on the following aspects: 1) controllable preparation of iron nanoparticles; 2) interface design of iron composites; 3) application of iron nanoparticles in heavy metals removal and electrocatalytic denitrification; 4) prospect of iron nanoparticles composites in environmental remediation.
\end{abstract}

Key words: iron nanoparticles; interface design; adsorption application; environmental electrocatalysis; environmental remediation; review

科学技术在推动社会快速发展的同时也不可避 免地会引发一系列环境问题，如废水/土壤中重金属 离子污染和持久性有机物污染等。近年来, 纳米铁 颗粒(Iron nanoparticles, 又称为纳米零价铁)因具有
低成本、高性能、无毒性及易回收等特性被广泛应 用于环境修复 ${ }^{[1-9]}$, 特别是作为还原剂/催化剂对重 金属离子、有机污染物(硝基芳香族化合物)及氧阴 离子(硝酸盐)等表现出良好的去除和降解能力。纳

收稿日期: 2020-06-24; 收到修改稿日期：2020-07-24; 网络出版日期：2020-09-09

基金项目: 霍英东青年教师基金(171041) Fok Ying-Tong Education Foundation of China (171041)

作者简介: 苏 莉(1988-), 女, 博士研究生. E-mail: 1169143@mail.dhu.edu.cn

SU Li(1988-), female, PhD candidate. E-mail: 1169143@mail.dhu.edu.cn

通信作者: 杨建平，教授. E-mail: jianpingyang@dhu.edu.cn

YANG Jianping, professor. E-mail: jianpingyang@dhu.edu.cn 
米铁颗粒的制备方法主要分为物理法和化学法, 但 通常粒径较小、表面能高, 由于本征磁性易产生团 聚, 降低了反应活性和稳定性 ${ }^{[7,10-13]}$ 。纳米复合材料 界面设计是通过基体和增强体界面结合构筑复合材 料整体 ${ }^{[14-18]}$, 界面的结合形式影响复合材料的性能 (图 1) ${ }^{[19-23]}$ 。因此, 对纳米铁颗粒的界面进行可控设 计来构建铁基纳米复合材料是解决上述问题的有效 方法。

目前，核壳结构(Core-shell)是铁基纳米复合材 料主要的界面设计方法之一。核壳结构是以纳米铁 颗粒为核, 通过化学键或者其他作用力将另一种纳 米材料包覆在核外形成的空间隔离纳米结构 ${ }^{[24-26]}$ 。 在该结构中, 纳米铁颗粒与壳层形成完全接触的界面, 既防止纳米铁颗粒团聚, 又有效地防止被氧化 ${ }^{[27-33] 。}$ 然而, 完全封闭的纳米壳层会减少纳米铁颗粒与反 应底物的接触, 不利于界面反应。介孔材料是一类 多孔纳米材料(孔径在 2 50 nm), 该材料具有孔径 连续可调、孔道结构规则有序以及比表面积大等特

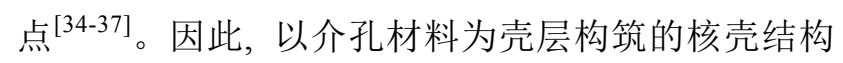
在改善纳米铁颗粒分散性和结构稳定性的同时也为 反应底物提供丰富的传输通道和吸附位点; 通过选 择性刻蚀等处理技术可以进一步得到中空的核壳结 构, 如典型的蛋黄结构(Yolk-shell), 核与壳之间将 不再形成紧密接触的界面, 并且具有协调可控的腔 体空间, 使得纳米核在腔内随意移动 ${ }^{[38-41]}$ 。这一

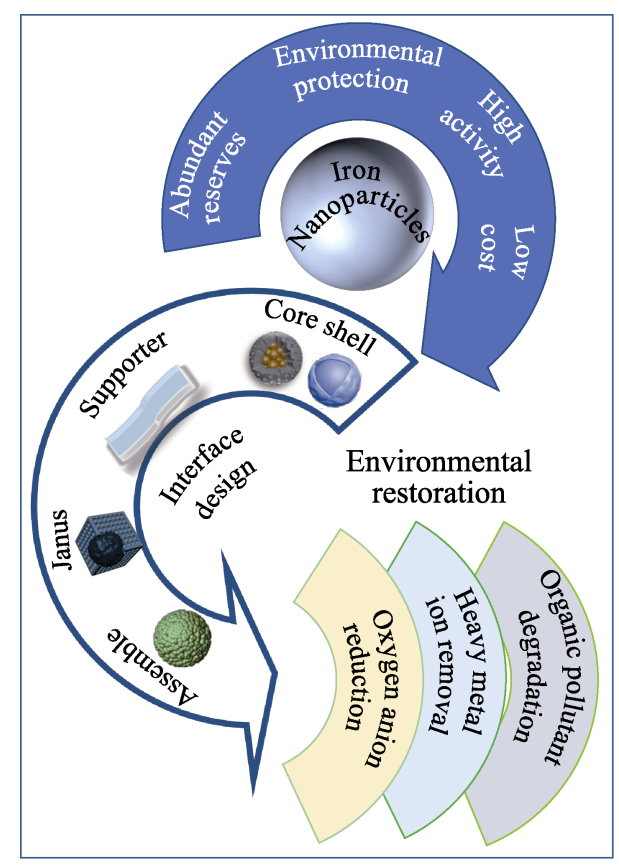

图 1 纳米铁颗粒的界面设计在环境修复中的应用

Fig. 1 Interface design of iron nanoparticles and applications in environmental remediation
结构增加了纳米铁颗粒表面活性位点的暴露程度, 可控的腔体也提供了相对独立的反应空间。尽管核 壳结构界面设计能有效地改善纳米铁颗粒的分散性 和提高结构的稳定性, 但这种完全包覆的界面设计 不利于纳米铁颗粒活性位点的暴露。

理想的多孔/介孔载体材料通常能够调控纳米 铁颗粒粒径, 提高纳米颗粒分散性和活性位点暴露 程度, 并提供定向的电荷传导路径 ${ }^{[2-44]}$ 。因此, 近年 来纳米铁颗粒负载于多孔/介孔纳米材料(Porous/ mesoporous materials, 如介孔 $\mathrm{C}, \mathrm{SiO}_{2}, \mathrm{Al}_{2} \mathrm{O}_{3}, \mathrm{TiO}_{2}$ 等) 的载体界面设计被广泛用于环境修复, 如电催化还 原硝酸根 ${ }^{[45-46]}$ 。目前, 非对称纳米结构和纳米颗粒 载体界面组装设计用于优化和实现纳米铁颗粒活性暴 露位点的精确调控, 增加复合材料中的金属含量 ${ }^{[47-49]}$ 。

本文着重总结纳米铁颗粒的制备方法并详细阐 述不同的纳米铁颗粒的界面设计, 以及界面结构在 重金属的富集和硝酸根的去除转化等方面的应用。

\section{1 纳米铁颗粒的合成方法}

\section{1 物理法}

物理法具有操作简便和成本低的特点。机械球 磨是通过研磨铁粉形成纳米级颗粒，该方法无毒、 高产, 但通常耗能高、产品纯度低。物理气相沉积 方法则可有效地提升产品纯度, 且制备的纳米颗粒 粒径更为均匀, 但是该方法能耗高, 安全性低, 不 适合量产。通常商业制备的纳米零价铁表面会被氧 化形成一层无定形的氧化铁, 针化了反应活性。近 期, Zhang 等 ${ }^{[50]}$ 提出了利用液氮作为超低温介质以 活化商用纳米铁颗粒(图 2(A)), 由于金属铁的热膨 胀系数远大于铁氧化物, 因此在快速冷却过程中引 起收缩应力, 导致断裂。测试表明液氮处理的纳米铁 颗粒表面的氧化层会出现一些平均深度约为 $200 \mathrm{~nm}$ 的裂缝, 元素的分布变得不均匀, 裂缝内部的铁浓 度明显高于裂缝外部, 而氧则相反。这些裂缝为反 应过程中 $\mathrm{H}_{2} \mathrm{O} / \mathrm{O}_{2}$ 转移至铁核提供了有效通道。

\section{2 化学法-团簇沉淀法}

化学法是制备高纯度、小粒径和高活性纳米铁 颗粒的重要方法。早期, You 等 ${ }^{[51]}$ 开发纳米团簇沉积 系统用于制备纳米铁颗粒(图 2(B)), 团簇中最初形 成的纯纳米铁颗粒沉积于基底, 随后将其暴露于空 气环境中会使每个纳米铁颗粒表面形成氧化层。通 过改变氦氩比例调控团簇腔内压力和生长距离, 形 


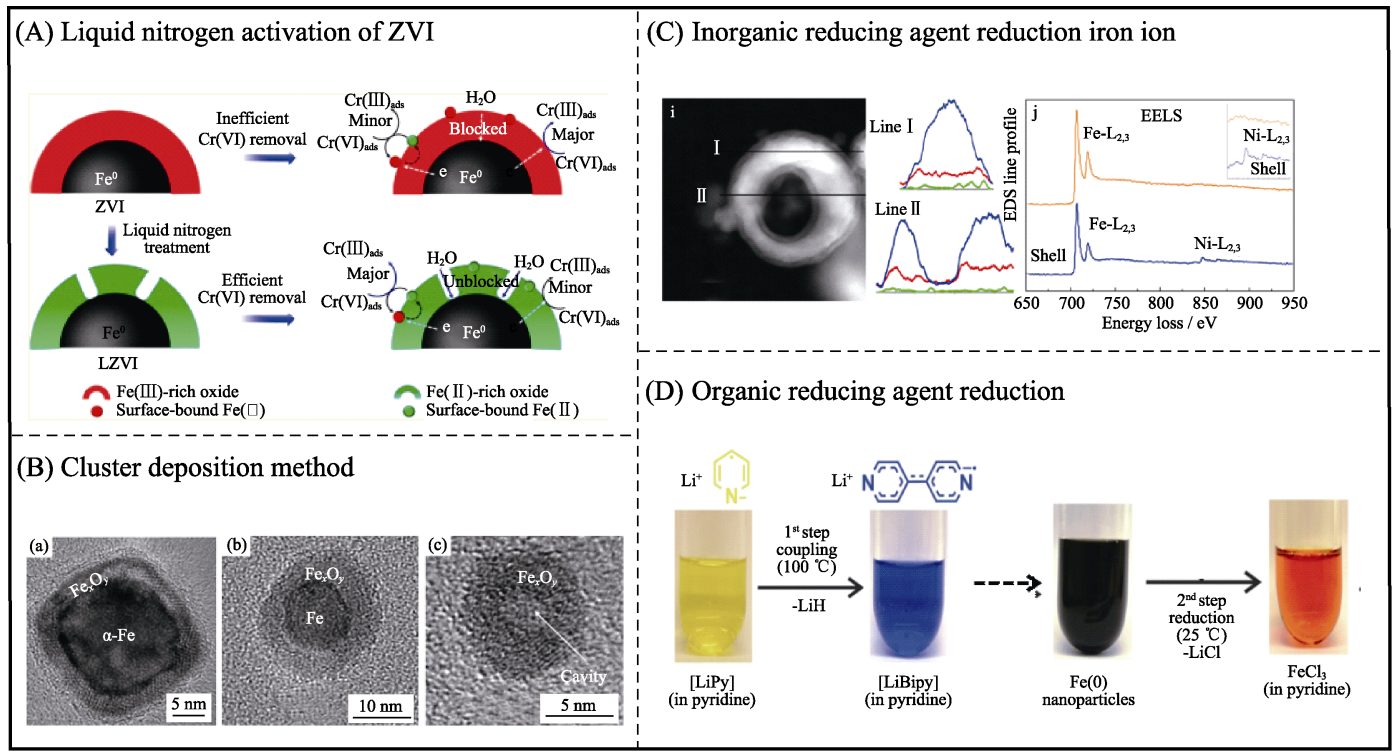

图 2 (A)液氮活化商业纳米铁颗粒 ${ }^{[50]},(\mathrm{B})$ 具有核壳结构的 $\mathrm{Fe}$ 纳米粒子 ${ }^{[51]},(\mathrm{C})$ 嗍氢化钠还原 $\mathrm{Fe}^{3+}$ 为纳米铁颗粒 ${ }^{[52]},(\mathrm{D})$ 有机还原剂两步还原铁离子为纳米铁颗粒 ${ }^{[55]}$

Fig. 2 (A) Liquid nitrogen activation of $\mathrm{ZVI}^{[50]}$, (B) HRTEM images showing three types of Fe nanoparticles with core-shell structure ${ }^{[51]}$, (C) sodium borohydride being introduced to reduce $\mathrm{Fe}^{3+}$ to $\mathrm{ZVI}^{[52]}$, (D) illustration of the [LiBipy]-driven two-step synthesis: formation of [LiBipy] radical by coupling reaction ${ }^{[55]}$

成 2 100 nm 的单分散纳米铁颗粒。通过石英微天 平, 沉积速率可调控至 $10 \mathrm{mg} / \mathrm{h}$ 。用 TEM 可观察到 $\alpha-\mathrm{Fe}$ 相的外层形成了一层铁氧化物。

\section{3 化学法-无机还原剂还原高价铁离子}

还原法是常用的化学法制备纳米铁的方法之 一。例如, Zhang 等 ${ }^{[52]}$ 采用滴定法利用硼氢化钠还 原 $\mathrm{Fe}^{3+}$ 得到球形的纳米铁颗粒，相应的反应方程式为:

$$
\begin{aligned}
& 4 \mathrm{Fe}^{3+}+3 \mathrm{BH}_{4}{ }^{-}+9 \mathrm{H}_{2} \mathrm{O} \rightarrow \\
& 4 \mathrm{Fe}^{0}+3 \mathrm{H}_{2} \mathrm{BO}_{3}{ }^{-}+12 \mathrm{H}^{+}+6 \mathrm{H}_{2}
\end{aligned}
$$

主要为 1 100 $\mathrm{nm}$ 的 bcc 相 Fe 纳米颗粒, 少数可达 到 200 250 nm。不同粒径的纳米铁颗粒与 $\mathrm{H}_{2} \mathrm{O} / \mathrm{O}_{2}$ 发生氧化反应形成相似的表面层, 测定和计算表明 该层为 2 4 nm 厚的无定形的 $\mathrm{FeOOH}$ (图 2(C))。随 后, $\mathrm{Wu}$ 等 ${ }^{[53]}$ 利用过量的硼氢化钠还原 $\mathrm{Fe}^{2+}$ 制得纳米 铁颗粒，相应反应方程式为:

$$
\mathrm{Fe}^{2+}+2 \mathrm{BH}_{4}{ }^{-}+6 \mathrm{H}_{2} \mathrm{O} \rightarrow \mathrm{Fe}^{0}+2 \mathrm{~B}(\mathrm{OH})_{3}+7 \mathrm{H}_{2}
$$

硫化是近期发展起来的一种稳定纳米铁颗粒的 新方法。目前硫化的方法主要是基于液相, 通过改 变前驱体种类、硫化时间和负载量来调控纳米铁颗 粒的硫化程度。Wu 等 ${ }^{[53]}$ 首先在 $\mathrm{FeSO}_{4}$ 体系中调控 $\mathrm{S}$ 源和 $\mathrm{Fe}$ 源的一系列比例, 随后通过滴定对新鲜纳米 铁颗粒进行硫化得到粒径 200 $\mathrm{nm}$, 纳米片包覆的 $\mathrm{S}-\mathrm{nZVI}$ 球形纳米颗粒。当 $\mathrm{S} / \mathrm{Fe}$ 比例从 0 增加至 0.35 后, 纳米片表面粗䊁程度明显增加, 这可能是因为形 成了 $\mathrm{FeS}$ 。 $\mathrm{EDS}$ 测试表明 $\mathrm{S} / \mathrm{Fe}$ 在纳米片中的比值为 0.26 , 纳米核为 0.1 , 即纳米片主要为 $\mathrm{FeS}$ 。新鲜的
S-nZVI 需要在 $4{ }^{\circ} \mathrm{C}$ 保存于乙醇溶液中, 防止被氧化。

\section{4 化学法-金属有机还原剂还原铁离子}

$\mathrm{Fe}(\mathrm{CO})_{5}$ 在离子液体中的热分解是制备高纯度 且粒径均匀的纳米铁颗粒的有效方法之一，但 $\mathrm{Fe}(\mathrm{CO})_{5}$ 剧毒且易燃 ${ }^{[54]}$ 。Feldmann 等 ${ }^{[55]}$ 以安全的金 属有机双吡啶 [LiBipy]为强还原剂合成纳米铁颗粒。 首先在吡啶中溶解 $\mathrm{Li}$, 可以观察到黄色的液相, 表 明形成了吡啶基自由基（[LiPy]）, 随后黄色溶液迅 速变为蓝色溶液 $(<5 \mathrm{~min})$ 。将溶液加热至 $100{ }^{\circ} \mathrm{C}$ 使 $\mathrm{Li}$ 全部溶解, 得到深蓝色的溶液, 表明生成了双吡 啶自由基([LiBipy]), 随后向溶液中注入 $\mathrm{FeCl}_{3}$ 吡啶 溶液, 瞬间会形成黑色纳米铁颗粒悬浮物(粒径为 3 5 nm), 纯化后可分散在吡啶或正庚烷中得到稳 定的悬浮液, 若添加油酸作为稳定剂, 则悬浮液可 以保持数月。将纳米铁颗粒悬浮液在真空中干燥可 得到粉末, 若置于空气中会表现出缓慢的氧化和脱 色, 且具有极高的反应活性, 在有氧的条件下会瞬 间着火(图 2(D))。

\section{5 化学法-微乳液法}

微乳液法通常是在有机相、水相、表面活性剂 和助剂存在的混合体系中制备。一般地, 将含有铁 盐的微乳液与含有还原剂的微乳液进行混合, 经过 乳化后形成粒径在 5 100 $\mathrm{nm}$ 的小液滴, 其间由于 胶团的接触碰撞, 水相液滴内的反应物会发生化学 反应，即调控制备得到不同粒径的纳米铁颗粒 ${ }^{[56}$ 。但 是，该方法制备成本较高，并且制备的新鲜纳米铁颗 

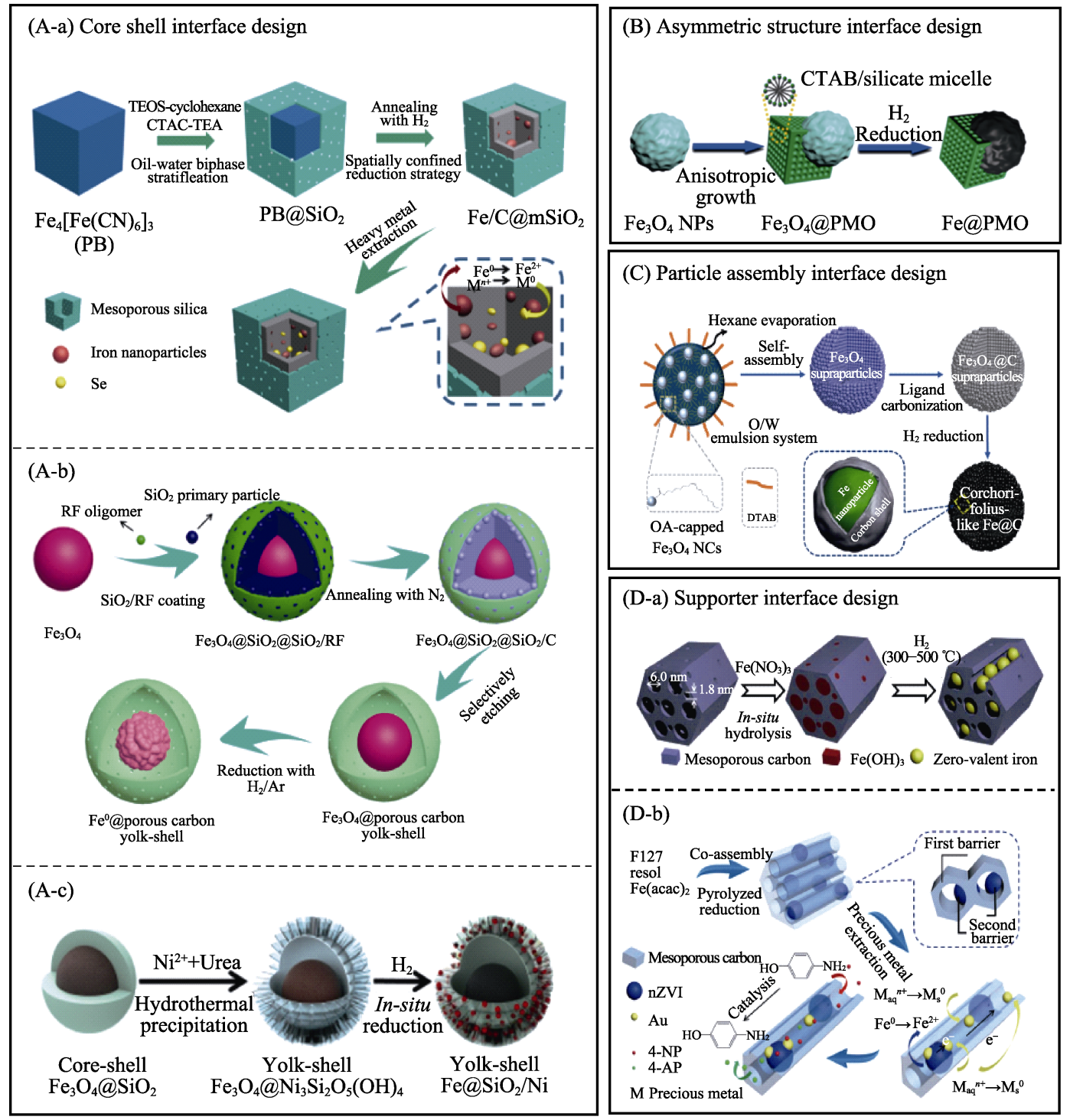

(D-a) Supporter interface design

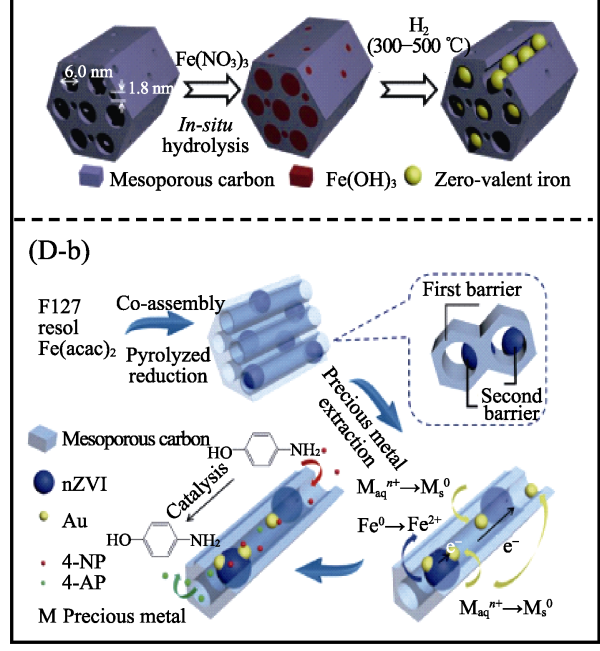

图 3 (A-a)胶囊状核壳结构 $\mathrm{Fe} / \mathrm{C} @ \mathrm{mSiO}_{2}$ 的制备示意图 ${ }^{[58]},(\mathrm{A}-\mathrm{b})$ 用溶胶一凝胶和原位热还原法构建蛋黄结构 $\mathrm{Fe}^{0} @ \mathrm{mC}$ 的制备示意图 ${ }^{[47]}$, $(\mathrm{A}-\mathrm{c})$ 分层蛋黄结构 $\mathrm{Fe} @ \mathrm{SiO}_{2} / \mathrm{Ni}$ 的制备路线图 ${ }^{[59]},(\mathrm{B}) \mathrm{Janus}$ 结构的 $\mathrm{Fe} @ \mathrm{PMO}$ 的合成示意图 ${ }^{[48]},(\mathrm{C})$ 类山莓状 CL-Fe@C 的合成示意

图 ${ }^{[49]},(\mathrm{D}-\mathrm{a})$ 纳米复合材料 $\mathrm{nZVI@OMC}$ 的合成路线图 ${ }^{[46]},(\mathrm{D}-\mathrm{b})$ 纳米铁颗粒负载于介孔碳(nZVI@C)的合成示意图 ${ }^{[65]}$

Fig. 3 (A-a) Schematic illustration of the small iron nanoparticles in a capsule $\left(\mathrm{Fe} / \mathrm{C} @ \mathrm{mSiO}_{2}\right)^{[58]}$, (A-b) schematic illustration of the Sol-Gel coating process and in-situ confined thermal reduction strategy for the fabrication of the porous carbon capsulated $\mathrm{Fe}^{0}$ yolk-shell nanospheres $\left(\mathrm{Fe}^{0} @ \mathrm{mC}\right)^{[47]}$, (A-c) illustration of the synthesis of hierarchical yolk-shell $\mathrm{Fe} @ \mathrm{SiO}_{2} / \mathrm{Ni}$ nanocomposites ${ }^{[59]}$,

(B) schematic illustration of the synthetic procedure for Fe@PMO with Janus structure ${ }^{[48]}$, (C) schematic illustration of in situ confined thermal reduction strategy for preparation of corchorifolius-like structure carbon-coated Fe microspheres $(\mathrm{CL}-\mathrm{Fe} @ \mathrm{C})^{[49]}$, (D-a) schematic illustration of the nZVI@OMC ${ }^{[46]}$,

(D-b) schematic of nanoscale zero-valent iron in mesoporous carbon (nZVI @C $)^{[65]}$

粒易于团聚导致活性下降。因此，对新鲜的纳米铁 颗粒进行表面涂层和乳化是有效提高纳米铁颗粒稳 定性的重要手段。表面涂层是在纳米铁颗粒表面修 饰官能团来改变颗粒表面的电荷状况, 进而改善其 分散性和稳定程度 ${ }^{[57]}$ 。此外, 基于不同类型的乳化 液乳化纳米铁颗粒(如油包水(Oil in Water, O/W)), 将 纳米铁颗粒置于非极性溶剂中可有效防止团聚 ${ }^{[58]}$ 。

相对于物理法的低成本和量级生产的特点, 化 学法则具有合成纳米铁纯度高、粒径更均匀的优势。
但两种方法制备的纳米铁颗粒都会由于高的表面活 性和本征磁性而发生不同程度的聚集，或者吸附到 其他物质表面，导致活性位点的暴露程度减少，有 效活性降低。因此, 有必要研究纳米铁颗粒复合材料。

\section{2 纳米铁颗粒复合材料的界面设计}

目前，已有大量的研究报道了纳米铁颗粒复合 材料的界面结构, 包括核壳结构、非对称结构、载 
体界面结构和组装结构等。这些界面结构可以防止 纳米铁颗粒团聚, 且可作为纳米微反应器提供相对 稳定的独立反应空间; 有效调控活性位点的暴露程 度; 有助于提高纳米复合材料中铁的含量; 有效改 善材料的结构稳定性和导电性。

\section{1 核壳结构}

核壳结构是指以纳米铁颗粒为核，外表面包覆 另一种纳米材料的壳层完全接触界面结构 ${ }^{[59-60]}$ 。

Yang 等 ${ }^{[88]}$ 基于空间限域热还原策略通过油水 两相法制备核壳结构的 $\mathrm{Fe} @ \mathrm{C} @ \mathrm{mSiO}_{2}$ (图 3(A-a))。 普鲁士蓝( $(\mathrm{PB})$ 纳米立方块热还原转变为 $\mathrm{Fe} @ \mathrm{C}, \sim 10 \mathrm{~nm}$ $\mathrm{Fe}$ 颗粒均匀分布在内部具有微小空隙的立方腔体 中, 发散树枝状介孔 $\mathrm{SiO}_{2}$ 壳层能使核壳结构依旧保 持立方形形貌。不同于实心的核壳结构, 蛋黄结构 则是由一个内部可移动的纳米铁颗粒核、空腔和外 部单层/多层纳米材料组成。协调可控的腔体可作为 微反应空间, 多孔/介孔壳层可提供反应所需的传输 通道和吸附位点。随后, Yang 等 ${ }^{[47]}$ 利用溶胶-凝胶法, 以球形 $\mathrm{Fe}_{3} \mathrm{O}_{4}$ 为核, 与碳源、硅源共水解和热还原 实现了 $\mathrm{SiO}_{2}$ 层和 $\mathrm{C}$ 层的依次包覆, 并通过选择性刻 蚀 $\mathrm{SiO}_{2}$ 和原位还原制备高度分散的蛋黄结构 $\mathrm{Fe}^{0} @ \mathrm{mC}$, 平均粒径为 $380 \mathrm{~nm}$, 空腔 $160 \mathrm{~nm}$ (图 3(A-b))。构 建介孔壳层有效增加了纳米颗粒的比表面积, 可达 $305 \mathrm{~m}^{2} / \mathrm{g}$, 孔径为 $0.8 \sim 15 \mathrm{~nm}$ 。

Jiao 等 ${ }^{[59]}$ 报道了蛋黄结构的 $\mathrm{Fe} @ \mathrm{SiO}_{2} / \mathrm{Ni}$ (图 $3(\mathrm{~A}-\mathrm{c})$ )。首先刻蚀 $\mathrm{SiO}_{2}$ 壳层并同时沉积超薄硅酸镍 纳米片, 将核壳结构的微球转化为以 $\mathrm{Fe}_{3} \mathrm{O}_{4}$ 核和硅 酸镍纳米片为壳的层级蛋黄结构 $\mathrm{Fe}_{3} \mathrm{O}_{4} @ \mathrm{SiO}_{2}$ 。随后 经过氢气还原处理得到蛋黄结构的 $\mathrm{Fe} @ \mathrm{SiO}_{2} / \mathrm{Ni}(\mathrm{Fe}$ 纳米核和 $\mathrm{SiO}_{2}$ 超薄纳米片组装的壳层, 其中高度分 散的 $\mathrm{Ni}$ 纳米颗粒基于原位自模板还原嵌入 $\mathrm{SiO}_{2}$ 纳 米片中), 平均厚度约为 $2.6 \mathrm{~nm}$ 的 $\mathrm{SiO}_{2}$ 纳米片上分 散着粒径约为 $4 \mathrm{~nm}$ 的 $\mathrm{Ni}$ 颗粒, 以此为反应的活性 位点，纳米片之间的空腔和层间间隙为反应通道。

\section{2 非对称结构}

纳米铁颗粒完全封装在限域空间的界面设计虽 然能够有效防止团聚, 但纳米铁颗粒活性位点的暴 露程度往往不可调控。因此, 设计非对称结构界面 设计, 如 Janus 结构对调控纳米粒子活性位点的暴 露程度具有重要意义 ${ }^{[53,62]}$ 。最近, Yang 等 ${ }^{[48]}$ 基于岛 状各向异性成核生长方法, 首先通过调控反应溶剂 比例以及有机硅的剂量制得一系列表面暴露程度不 同的 $\mathrm{Fe}_{3} \mathrm{O}_{4} @ \mathrm{PMO}$, 随后在 $\mathrm{H}_{2}$ 中原位热还原得到 $\mathrm{Fe} @ \mathrm{PMO}$, 仍保持原始形貌(图 3(B)), 由于 $\mathrm{Fe}_{3} \mathrm{O}_{4}$ 还 原过程中 $\mathrm{O}$ 的脱除使制得的 $\mathrm{Fe}$ 纳米微球是由不规
则、小粒径的 $\mathrm{Fe}$ 颗粒组成。当增加合成体系中有机 硅烷的剂量和反应溶剂中乙醇的体积比时, 非对称 的 Janus 结构会分别逐渐演变为中心对称的立方形 和球形核壳结构，这表明 $\mathrm{PMO}$ (周期性有机介孔二 氧化硅)在 $\mathrm{Fe}_{3} \mathrm{O}_{4}$ 纳米颗粒表面的生长方式由异相成 核逐渐转变为均相成核, 单颗粒间的组装结构则由 非对称的 Janus 结构逐渐演变为对称的核壳结构。 其中, Janus 结构 Fe@PMO 中球形 $\mathrm{Fe}$ 颗粒可被针定 在立方 PMO 的一角或棱上, 调控合成条件使 $1 / 4 \sim 3 / 4$ 的 $\mathrm{Fe}$ 表面直接暴露, 在反应过程中可与催化底物直 接接触。

\section{3 纳米颗粒的组装}

相较于构筑特殊的壳层界面结构, 基于自组装一 热还原方法的纳米颗粒界面组装设计可用于制备高 分散性、高金属含量的铁基组装纳米复合材料。 $\mathrm{Su}$ 等 ${ }^{\left[{ }^{[9]}\right.}$ 以类山莓状碳包覆纳米铁颗粒组装的微球 $(\mathrm{CL}-\mathrm{Fe} @ \mathrm{C})$ 作为电催化反硝化脱氮的阴极材料 (图 3(C))。以形貌均一、粒径约为 $9 \mathrm{~nm}$ 的球形 $\mathrm{OA}-\mathrm{Fe}_{3} \mathrm{O}_{4}$ 纳米粒子 $(\mathrm{OA}$, 油酸)为超晶格的构筑单 元, 基于自组装方法制备长程有序的超晶格微球 $\mathrm{OA}-\mathrm{Fe}_{3} \mathrm{O}_{4}$, 随后, 通过配体碳化和原位热还原处理 制得由单颗粒径约为 $20 \mathrm{~nm}$ 的碳包覆纳米铁颗粒组 装的 CL-Fe@C 微球, 粒径为 $200 \mathrm{~nm} 2 \mu \mathrm{m}$, 且表面 均匀包覆了一层约为 $5 \mathrm{~nm}$ 的碳层。纳米铁颗粒在热 还原处理后粒径的增大归因于在 $\mathrm{Fe}_{3} \mathrm{O}_{4}$ 相转变为 $\mathrm{Fe}$ 相的过程中 $\mathrm{O}$ 逸出导致相邻纳米粒子发生了团聚, 而碳壳的空间阻隔使晶体未能继续生长。由于 $\mathrm{OA}$ 修饰在 $\mathrm{Fe}_{3} \mathrm{O}_{4}$ 表面的数量是固定的, 所以制得的 $\mathrm{CL}-\mathrm{Fe} @ \mathrm{C}$ 中 $\mathrm{Fe}$ 含量要比 $\mathrm{C}$ 含量高, 这将为制备高 金属含量催化剂提供新思路。

\section{4 载体}

碳基材料如介孔碳、石墨烯等具有大的比表面 积、良好的化学稳定性及导电性, 是用于负载纳米 铁颗粒的良好载体 ${ }^{[63-66]}$ 。纳米铁颗粒与载体之间的 界面和载体的骨架都能有效地传输电子。Zhang 等[46] 基于后合成方法制备了粒径 3 9 $\mathrm{nm}$ 的铁颗粒负载 在有序介孔碳 nZVI@OMC 中, 并通过改变铁源剂 量和热还原温度调控 $\mathrm{nZVI@OMC}$ 中 $\mathrm{Fe}$ 的含量 (0 45\%)。由于采用了 p $6 \mathrm{~m}$ 空间群的六方晶系有序介 孔碳，这使复合材料的表面积增大，可达 660 $830 \mathrm{~m}^{2} / \mathrm{g}$ (图 3(D-a))。Teng 等 ${ }^{[65]}$ 基于介孔碳骨架原位生长了 $\mathrm{Fe}_{3} \mathrm{O}_{4}$ 纳米颗粒, 通过原位热还原得到了由粒径约 为 $16 \mathrm{~nm}$ 的铁颗粒负载于介孔碳骨架的纳米复合 材料 nZVI@C (图 3(D-b))。

近年来, 纳米铁颗粒的界面设计能够有效地解 
决纯纳米铁颗粒易团聚、被氧化的问题, 并且纳米 材料的结构稳定性也得到了提高, 这些都有益于提 升纳米铁颗粒在环境修复中的还原/催化性能。

\section{3 纳米铁颗粒界面复合材料在环境修 复中的应用}

\section{1 重金属离子的回收及利用}

纳米铁颗粒界面复合材料去除重金属离子的机 理主要有吸附、还原和沉淀/共沉淀, 如表面形成重 金属离子的氢氧化物或氧化物 ${ }^{[68-70]}$ 。例如, Zhang 等 ${ }^{[50]}$ 用 $77 \mathrm{~K}$ 的液氮活化商用纳米铁颗粒可使其表面大 量的氧化铁壳层发生破裂, 这些破裂的缝隙为 $\mathrm{Cr}(\mathrm{VI})$ 提供了传质通道, $\mathrm{H}_{2} \mathrm{O} / \mathrm{O}_{2}$ 可扩散进入 $\mathrm{Fe}$ 核的 位置, 与 $\mathrm{Fe}$ 核反应后原位生成具有还原性的 $\mathrm{Fe}$ (II) 物种(图 4(A-a))。结果表明, 活化后的纳米铁颗粒在 去除 $\mathrm{Cr}(\mathrm{VI})$ 的过程中于 $\mathrm{Fe}$ 颗粒表面原位生成 $\mathrm{Fe}(\mathrm{III}) / \mathrm{Cr}(\mathrm{III}) / \mathrm{Cr}(\mathrm{VI})$, 去除 $\mathrm{Cr}(\mathrm{VI})$ 的同时避免了二 次污染。Zhang 等 ${ }^{[53]}$ 利用硫化修饰得到了核壳结构 的 S-nZVI, 用于去除污水中的 As(III) (图 4(B))。在 这个反应中, 氧气破坏了核壳结构, 并通过硫化铁 $(\mathrm{FeS})$ 转化生成氧化剂, 促进氧化 $\mathrm{As}(\mathrm{III})$ 。

载体界面设计中, Zhang 等 ${ }^{[69]}$ 将纳米铁颗粒负 载在类石墨烯的 $\mathrm{g}-\mathrm{C}_{3} \mathrm{~N}_{4}$ 纳米片上, 形成 $\mathrm{g}-\mathrm{nZVI}$ 纳 米复合材料, 用于去除重金属 $\mathrm{Pb}(\mathrm{II})$ (图 4(C))。用
g-nZVI 处理 $50 \mathrm{~L} 、 10 \mathrm{mg} / \mathrm{L}$ 的 $\mathrm{Pb}(\mathrm{II})$, 循环 5 次后 的去除率大大超过纯纳米铁颗粒对 $\mathrm{Pb}(\mathrm{II})$ 的去除率, 并且能够还原得到更多的金属 $\mathrm{Pb}$ 。

Yang 等 ${ }^{[47]}$ 设计了腔体空间可调控的蛋黄结构 $\mathrm{Fe}^{0} @ \mathrm{mC}(\mathrm{mC}$, 介孔碳壳层)用于去除水体系中的重 金属离子(如 $\mathrm{Au}(\mathrm{III})), 3 \mathrm{~h}$ 的去除率可接近 100\%。磁 分离回收的 $\mathrm{Fe}^{0} @ \mathrm{mC}-\mathrm{Au}$ 用于降解有机污染物 4-硝 基苯酚催化剂, 展现出良好的催化活性。这种介孔 壳层完全包覆的界面设计充分体现了去除一回收一 体的环保理念。此外, Yang 等 ${ }^{[48]}$ 设计的非对称 Janus 结构 Fe@PMO 对水体系中的 $\mathrm{Au}(\mathrm{III})$ 表现出快速吸 附还原的效果, 在反应前 $10 \mathrm{~min}$ 的去除率可达 $71 \%$, $3 \mathrm{~h}$ 后可达 98\% (图 4(D))。 Tafel 和 Zeta 测试表明, Janus 结构 $\mathrm{Fe} @ \mathrm{PMO}$ 中直接暴露的 $\mathrm{Fe}$ 表面更易被 反应体系中的氢质子和 $\mathrm{Au}(\mathrm{III})$ 腐蚀, 原位形成具有 还原性的活性中间体 $\mathrm{Fe}(\mathrm{II})$, 成为 $\mathrm{Au}(\mathrm{III})$ 的主要吸 附和沉积位点。非对称界面结构设计将为重金属的 快速回收提供新的解决方法。

\section{2 硝酸根的去除转化}

氮元素污染地下水的主要形式是硝酸盐污染, 硝酸盐具有强的化学稳定性, 且环境自净周期长。电 催化还原硝酸根无需后处理，能耗低 ${ }^{[71-72] 。}$

$\mathrm{Su}$ 等 ${ }^{[49]}$ 采用自组装和原位热还原法制得高铁 含量( 74\%)的类山莓状 CL-Fe@C 纳米微球, 用于 电催化高效去除硝酸根。研究表明, 在“Rate-determining step, 速率决速步” 中, 粗粘的微球表面有利于吸附体

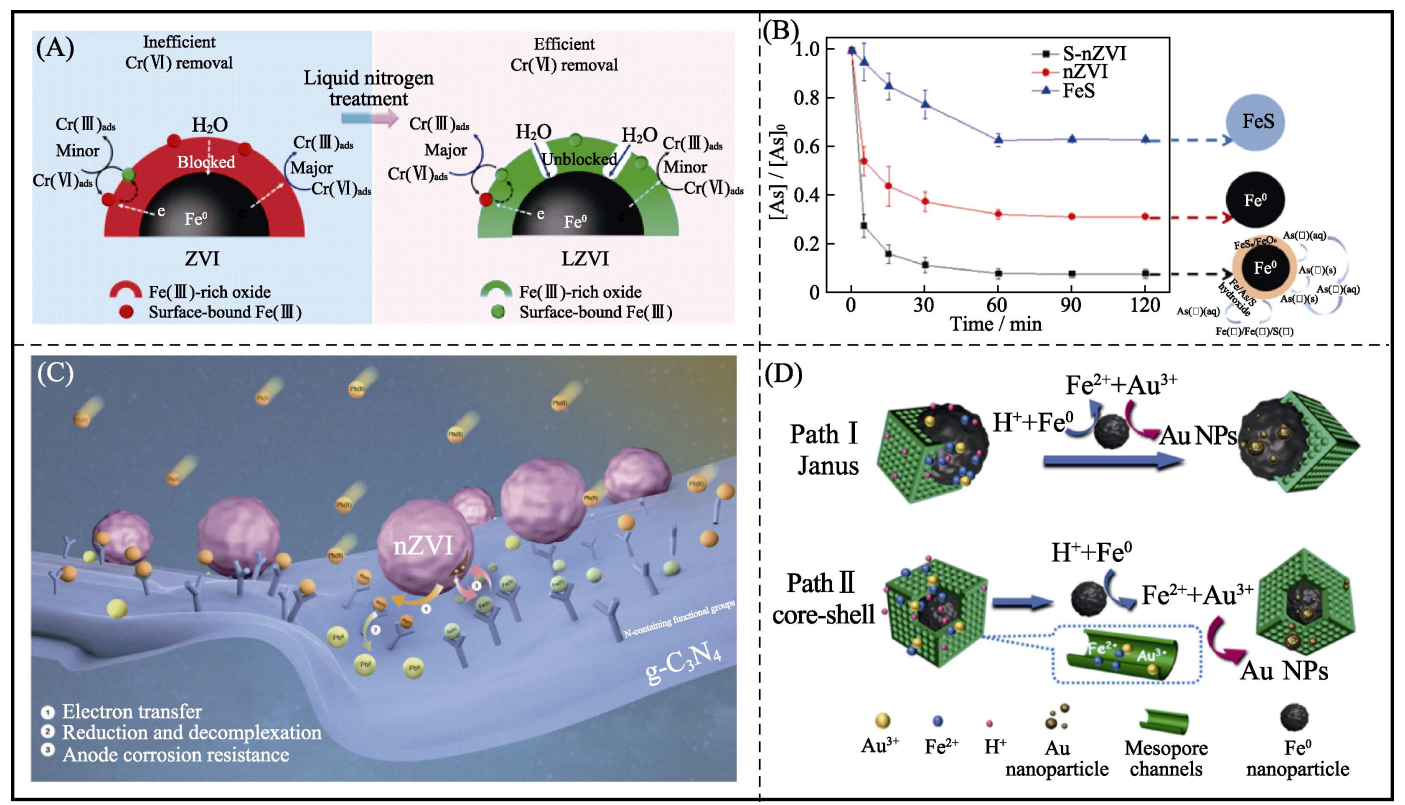

图 4 (A)液氮活化商用 ZVI 增强 $\mathrm{Cr}(\mathrm{VI})$ 去除能力的机理示意图 ${ }^{[50]},(\mathrm{B}) \mathrm{S}-\mathrm{nZVI}$ 对水溶液中 As(III) 的去除机理 ${ }^{[53]}$, (C) g-nZVI 对 $\mathrm{Pb}(\mathrm{II})$ 的去除机理 ${ }^{[69]}$, (D)Janus 和核壳结构 Fe@PMO 的还原金属离子机理图 ${ }^{[48]}$

Fig. 4 (A) Enhanced Cr(VI) removal mechanism of liquid nitrogen treated ZVI ${ }^{[50]}$, (B) S-nZVI for As(III) removal from aqueous solutions ${ }^{[53]}$, (C) proposed mechanism of $\mathrm{Pb}(\mathrm{II})$ removal using the g-nZVI ${ }^{[69]}$, (D) schematic diagram of the mechanism of metal ion reduced by Fe@PMO with Janus and core shell nanostructure ${ }^{[48]}$ 
系中硝酸根, 并有效地促使 $\mathrm{NO}_{3}{ }^{-}$转化为 $\mathrm{NO}_{2}{ }^{-}$; 而在 “Selectivity-determining step, 选择决速步”中, 高铁 含量能高效催化还原吸附在表面的 $\mathrm{NO}_{2}{ }^{-}$转化为中间 产物 $\mathrm{N}_{2} \mathrm{O}$, 生成目标产物 $\mathrm{N}_{2}$ (图 5(A))。在浓度为 $100 \mathrm{mg} / \mathrm{L}$ 体系中, $\mathrm{NO}_{3}{ }^{-}$的转化率接近 $100 \%, \mathrm{~N}_{2}$ 可达 到 $98 \%$ 。Feng 等 ${ }^{[71]}$ 基于载体界面设计制备了纳米铁 颗粒负载石墨烯 $\mathrm{Fe} @ \mathrm{NeC}$, 在还原硝酸根中展现出 良好的催化性能和循环稳定性, $\mathrm{Fe}(20 \%) @ \mathrm{NeC}$ 还原 $\mathrm{NO}_{3}{ }^{-}$的转化率为 $83 \%$ 。这种由纳米铁颗粒与载体形 成的界面可以有效地固定纳米颗粒，通过核壳结构 中电子从铁核向碳壳层的转移, 削弱了相邻碳原子 间的功函(图 5(B))。Zhang 等 ${ }^{[72]}$ 利用超薄的氮—碳纳 米片包覆氮掺杂的纳米铁颗粒, 形成了较高吡咯氮 含量 (17.4at \%) 和较大比表面积 $\left(2040 \mathrm{~m}^{2} / \mathrm{g}\right)$ 的类核壳结构 FeN-NC(图 5(C))。在去除硝 酸根的反应中， $\mathrm{N}_{2}$ 选择性可达 $91 \%$ ，去除硝 酸盐能力为 $6004 \mathrm{mg}(\mathrm{N}) / \mathrm{g}(\mathrm{Fe})$ 。通过调控 $\mathrm{NC}$ 壳层的厚度实现 $\mathrm{NO}_{3}{ }^{-}$到 $\mathrm{N}_{2}$ 多电子的转移。通过调控 $\mathrm{Fe}$ 基复合材料的界面, 实现了性能高、成本低和环
境影响小的电催化硝酸根的去除转换应用。

\section{4 结束语}

纳米铁颗粒的团聚是影响其环境修复应用的重 要问题之一, 去除能力降低与纳米铁颗粒表面活性 位点减少密切相关。因此, 纳米铁颗粒的界面设计 不仅抑制了团聚，提高了分散性，而且实现了纳米 铁颗粒表面活性位点的调控, 使其在环境修复中提 高了去除和降解能力，但是挑战依旧存在：

1) 真实环境中的污水往往同时具有多种污染 物，因此针对污水中同时存在的不同亲-疏水性的 多种类污染物 (无机污染物, 有机污染物), 通过优 化载体界面设计期待实现同步或逐步去除两种以上 的污染物;

2) 纳米复合材料中零价铁是提高其去除能力 的重要因素，因此基于颗粒组装界面设计精确调控 复合材料中铁含量, 对优化催化剂的催化性能具有 重要意义;

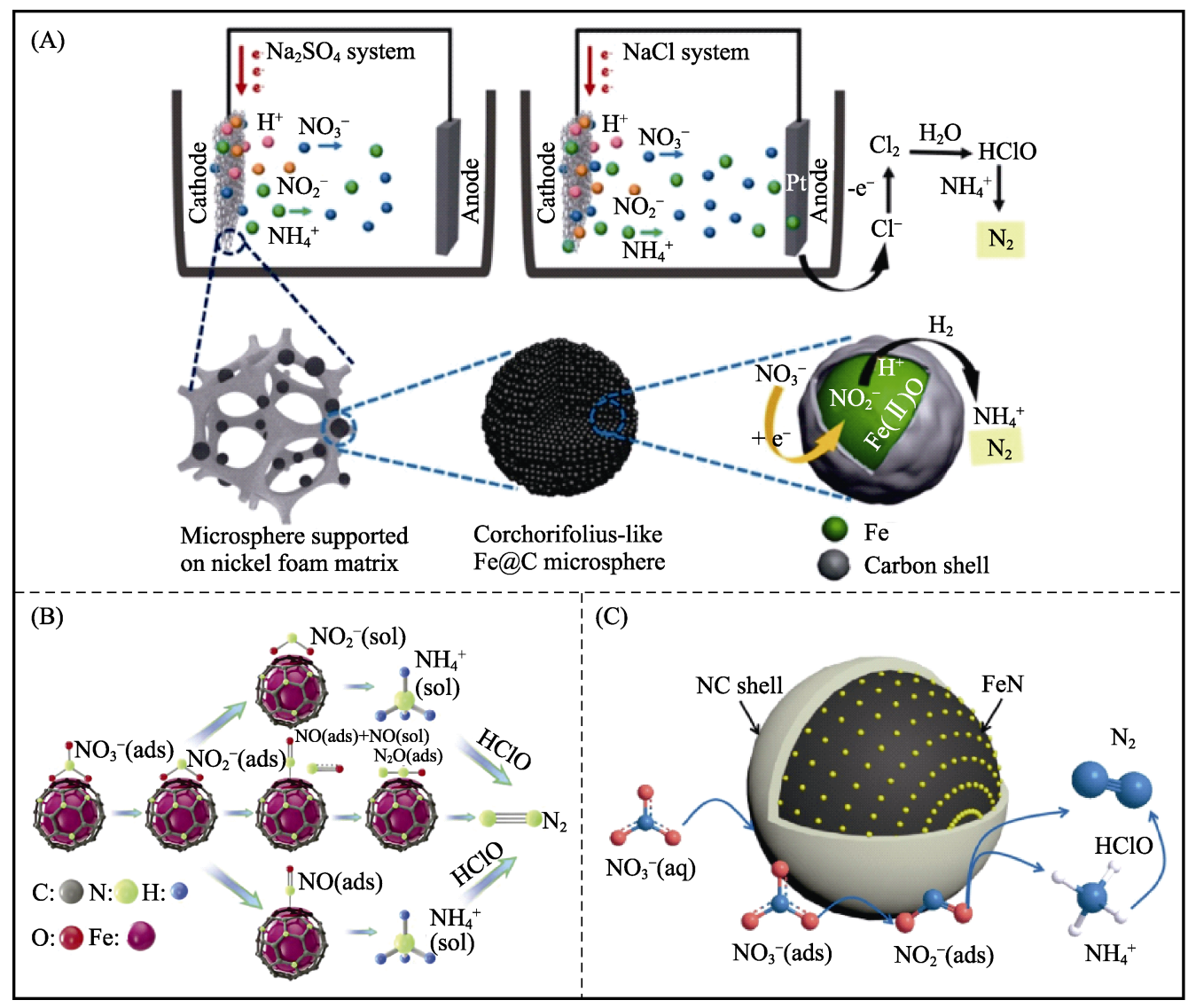

图 5 (A)不同电解液中 $\mathrm{CL}-\mathrm{Fe} @ \mathrm{C}$ 电催化还原硝酸根机理图 ${ }^{[49]},(\mathrm{B}) \mathrm{Fe} @ \mathrm{NeC}$ 电催化 还原硝酸根机理图 ${ }^{[71]},(\mathrm{C}) \mathrm{FeN}-\mathrm{NC}$ 催化还原硝酸根机理图 ${ }^{[72]}$

Fig. 5 (A) Scheme of the mechanism of electrocatalytic reduction of nitrate on the CL-Fe@C in a different electrolyte ${ }^{[49]}$, (B) proposed mechanism for NRR on $\mathrm{Fe} @ \mathrm{NeC}^{[71]},(\mathrm{C})$ reaction mechanism of FeN-NC for nitrate reduction ${ }^{[72]}$ 
3) 快速去除污染和富集，对节约成本和时间 具有现实意义, 精准调控复合材料中纳米铁颗粒表 面活性位点的暴露程度显得尤为重要, 因此需要研 究设计更为丰富的非对称界面结构(如 Janus 结构);

4) 随着科技的进步, 环境修复中污染的快速 检测逐渐采用便携式电子设备, 因此需要进一步地 开发和设计自支撑催化电极。

\section{参考文献:}

[1] LOWRY G V, JOHNSON K M. Congener-specific dechlorination of dissolved PCBs by microscale and nanoscale zerovalent iron in a water/methanol solution. Environmental Science \& Technology, 2004, 38(19): 5208-5216.

[2] WANG C B, ZHANG W X. Synthesizing nanoscale iron particles for rapid and complete dechlorination of TCE and PCBs. Environmental Science \& Technology, 1997, 31(7): 2154-2156.

[3] LI X Q, ZHANG W X. Iron nanoparticles: the core-shell structure and unique properties for $\mathrm{Ni}(\mathrm{II})$ sequestration. Langmuir, 2006, 22(10): 4638-4642.

[4] TENG W, BAI N, ZHANG W X, et al. Selective nitrate reduction to dinitrogen by electrocatalysis on nanoscale iron encapsulated in mesoporous carbon. Environmental Science \& Technology, 2018, 52(1): 230-236.

[5] PONDER S M, DARAB J G, MALLOUK T E. Remediation of $\mathrm{Cr}(\mathrm{VI})$ and $\mathrm{Pb}(\mathrm{II})$ aqueous solutions using supported, nanoscale zero-valent iron. Environmental Science \& Technology, 2000, 34(12): 2564-2569.

[6] FU F, DIONYSIOU D D, LIU H. The use of zero-valent iron for groundwater remediation and wastewater treatment: a review. Journal of Hazardous Materials, 2014, 267: 194-205.

[7] PHENRAT T, SALEH N, LOWRY G V, et al. Aggregation and sedimentation of aqueous nanoscale zerovalent iron dispersions. Environmental Science \& Technology, 2007, 41(1): 284-290.

[8] YANG Z, QIAN J, PAN B C, et al. Singlet oxygen mediated iron-based Fenton-like catalysis under nanoconfinement. Proceedings of the National Academy of Sciences, 2019, 116(14): 6659-6664.

[9] QIN H, GUAN X, TRATNYEK P G. Effects of sulfidation and nitrate on the reduction of N-nitrosodimethylamine by zerovalent iron. Environmental Science \& Technology, 2019, 53(16): 9744-9754.

[10] TOSCO T, CRUZ V C, SETHI R, et al. Nanoscale zerovalent iron particles for groundwater remediation: a review. Journal of Cleaner Production, 2014, 77: 10-21.

[11] HUA Y, WANG W, ZHANG W X, et al. Effect of bicarbonate on aging and reactivity of nanoscale zerovalent iron (nZVI) toward uranium removal. Chemosphere, 2018, 201: 603-611.

[12] GRIEGER K D, BJERG P L, BAUN A, et al. Environmental benefits and risks of zero-valent iron nanoparticles (nZVI) for in situ remediation: risk mitigation or trade-off? Journal of Contaminant Hydrology, 2010, 118(3): 165-183.

[13] ZHU F, LI L, LIU T, et al. Effect of $\mathrm{pH}$, temperature, humic acid and coexisting anions on reduction of $\mathrm{Cr}(\mathrm{VI})$ in the soil leachate by nZVI/Ni bimetal material. Environmental Pollution, 2017, 227: 444-450.

[14] HUANG W, LI W X. Surface and interface design for heterogeneous catalysis. Physical Chemistry Chemical Physics, 2019, 21(2): 523-536.

[15] CHU K, WANG F, ZHANG H, et al. Interface design of graphene/ copper composites by matrix alloying with titanium. Materials \& Design, 2018, 144: 290-303.

[16] CHEN P C, LIU M, MIRKIN C A, et al. Interface and heterostructure design in polyelemental nanoparticles. Science, 2019, 363(6430): 959.
[17] YANG Z, LIU J, WANG F, et al. Rational design of covalent interfaces for graphene/elastomer nanocomposites. Composites Science and Technology, 2016, 132: 68-75.

[18] CHANG W S, LIU H J, CHU Y H, et al. Tuning electronic transport in a self-assembled nanocomposite. ACS Nano, 2014, 8(6): 6242-6249.

[19] ESPINO P E, BRAS J, DOMENEK S, et al. Designed cellulose nanocrystal surface properties for improving barrier properties in polylactide nanocomposites. Carbohydrate Polymers, 2018, 183: 267-277.

[20] PENG J, CHENG Q. High-performance nanocomposites inspired by nature. Advanced Materials, 2017, 29(45): 1702959.

[21] HUANG J, TANG Z, GUO B, et al. Bioinspired interface engineering in elastomer/graphene composites by constructing sacrificial metalligand bonds. Macromolecular Rapid Communications, 2016, 37(13): 1040-1045.

[22] SANCHEZ C, RIBOT F, LEBEAU B. Molecular design of hybrid organic-inorganic nanocomposites synthesized via Sol-Gel chemistry. Journal of Materials Chemistry, 1999, 9(1): 35-44.

[23] ZHANG Y, GONG S, CHENG Q, et al. Graphene-based artificial nacre nanocomposites. Chemical Society Reviews, 2016, 45(9): 2378-2395.

[24] NALDONI A, PSARO R, DAL S V, et al. Effect of nature and location of defects on bandgap narrowing in black $\mathrm{TiO}_{2}$ nanoparticles. Journal of the American Chemical Society, 2012, 134(18): 7600-7603.

[25] TANG J, LIU J, IMURA M, et al. Thermal conversion of core-shell metal-organic frameworks: a new method for selectively functionalized nanoporous hybrid carbon. Journal of the American Chemical Society, 2015, 137(4): 1572-1580.

[26] XU Z C, HOU Y L, SUN S H. Magnetic core/shell $\mathrm{Fe}_{3} \mathrm{O}_{4} / \mathrm{Au}$ and $\mathrm{Fe}_{3} \mathrm{O}_{4} / \mathrm{Au} / \mathrm{Ag}$ nanoparticles with tunable plasmonic properties. Journal of the American Chemical Society, 2007, 129(28): 8698-8699.

[27] TSENG H H, SU J G, LIANG C. Synthesis of granular activated carbon/zero valent iron composites for simultaneous adsorption/ dechlorination of trichloroethylene. Journal of Hazardous Materials, 2011, 192(2): 500-506.

[28] LI Z, WANG L, MENG J, et al. Zeolite-supported nanoscale zerovalent iron: new findings on simultaneous adsorption of $\mathrm{Cd}(\mathrm{II})$, $\mathrm{Pb}(\mathrm{II})$, and $\mathrm{As}(\mathrm{III})$ in aqueous solution and soil. Journal of Hazardous Materials, 2018, 344: 1-11.

[29] LUO W, WANG Y, YANG J P, et al. Silicon/mesoporous carbon/ crystalline $\mathrm{TiO}_{2}$ nanoparticles for highly stable lithium storage. ACS Nano, 2016, 10(11): 10524-10532.

[30] LU W, LI J, CHEN L, et al. One-pot synthesis of magnetic iron oxide nanoparticle-multiwalled carbon nanotube composites for enhanced removal of $\mathrm{Cr}(\mathrm{VI})$ from aqueous solution. Journal of Colloid and Interface Science, 2017, 505: 1134-1146.

[31] YANG J P, ZHANG F, ZHAO D Y, et al. Large pore mesostructured cellular silica foam coated magnetic oxide composites with multilamellar vesicle shells for adsorption. Chemical Communications, 2014, 50(6): 713-715.

[32] YANG J P, DOU S X, ZHAO D Y, et al. Monodisperse core-shell structured magnetic mesoporous aluminosilicate nanospheres with large dendritic mesochannels. Nano Research, 2015, 8(8): 2503-2514.

[33] YANG J P, ZHANG F, ZHAO D Y, et al. Mesoporous silicacoated plasmonic nanostructures for surface-enhanced Raman scattering detection and photothermal therapy. Advanced Healthcare Materials, 2014, 3(10): 1620-1628.

[34] ZHAO D Y, HUO Q, STUCKY G D, et al. Nonionic triblock and star diblock copolymer and oligomeric surfactant syntheses of highly ordered, hydrothermally stable, mesoporous silica structures. Journal of the American Chemical Society, 1998, 120(24): 6024-6036.

[35] BECK J S, VARTULI J C, SCHLENKER J L, et al. A new family of mesoporous molecular sieves prepared with liquid crystal templates. Journal of the American Chemical Society, 1992, 114(27): 10834-10843. 
[36] INAGAKI S, GUAN S, TERASAKI O, et al. Novel mesoporous materials with a uniform distribution of organic groups and inorganic oxide in their frameworks. Journal of the American Chemical Society, 1999, 121(41): 9611-9614.

[37] LI W, ZHANG F, ZHAO D Y, et al. A versatile kinetics-controlled coating method to construct uniform porous $\mathrm{TiO}_{2}$ shells for multifunctional core-shell structures. Journal of the American Chemical Society, 2012, 134(29): 11864-11867.

[38] KAMATA K, LU Y, XIA Y. Synthesis and characterization of monodispersed core-shell spherical colloids with movable cores. Journal of the American Chemical Society, 2003, 125(9): 2384-2385.

[39] LI W, DENG Y H, ZHAO D Y, et al. Hydrothermal etching assisted crystallization: a facile route to functional yolk-shell titanate microspheres with ultrathin nanosheets-assembled double shells. Journal of the American Chemical Society, 2011, 133(40): 15830-15833.

[40] YUE Q, LI J, SU J, et al. Plasmolysis-inspired nanoengineering of functional yolk-shell microspheres with magnetic core and mesoporous silica shell. Journal of the American Chemical Society, 2017, 139(43): 15486-15493.

[41] SUN H, SHEN X, CHEN H, et al. Measuring the unusually slow ionic diffusion in polyaniline via study of yolk-shell nanostructures. Journal of the American Chemical Society, 2012, 134(27): 11243-11250.

[42] ANTOLINI E. Carbon supports for low-temperature fuel cell catalysts. Applied Catalysis B: Environmental, 2009, 88(1): 1-24.

[43] BANG J H, HAN K, SUSLICK K S, et al. Porous carbon supports prepared by ultrasonic spray pyrolysis for direct methanol fuel cell electrodes. The Journal of Physical Chemistry C, 2007, 111(29): 10959-10964.

[44] SKRABALAK S E, SUSLICK K S. Porous carbon powders prepared by ultrasonic spray pyrolysis. Journal of the American Chemical Society, 2006, 128 (39): 12642-12643.

[45] XU H W, ZHANG W X, YANG J P, et al. Bimetallic PdCu nanocrystals immobilized by nitrogen-containing ordered mesoporous carbon for electrocatalytic denitrification. ACS Applied Materials \& Interfaces, 2019, 11(4): 3861-3868.

[46] TENG W, BAI N, ZHANG W X, et al. Selective nitrate reduction to dinitrogen by electrocatalysis on nanoscale iron encapsulated in mesoporous carbon. Environ. Sci. Technol., 2018, 52(1): 230-236.

[47] WANG Q Q, ZHANG W Z, YANG J P, et al. Porous-carbonconfined formation of monodisperse iron nanoparticle yolks toward versatile nanoreactors for metal extraction. Chemistry-A European Journal 2018, 24(58): 15663-15668.

[48] SU L, JIAN W, YANG J P, et al. Site-selective exposure of iron nanoparticles to achieve rapid interface enrichment for heavy metals. Chemical Communications, 2020, 56(18): 2795-2798.

[49] SU L, JIAN W, YANG J P, et al. Tailoring the assembly of iron nanoparticles in carbon microspheres toward high-performance electrocatalytic denitrification. Nano Letters, 2019, 19(8): 5423-5430.

[50] HU Y, PENG X, ZHANG L, et al. Liquid nitrogen activation of zero-valent iron and its enhanced $\mathrm{Cr}(\mathrm{VI})$ removal performance. Environmental Science \& Technology, 2019, 53(14): 8333-8341.

[51] WANG C, BAER D R, QIANG Y, et al. Morphology and electronic structure of the oxide shell on the surface of iron nanoparticles. Journal of the American Chemical Society, 2009, 131(25): 8824-8832.

[52] LING L, ZHANG W X. Reactions of nanoscale zero-valent iron with $\mathrm{Ni}(\mathrm{II})$ : three-dimensional tomography of the "Hollow out" effect in a single nanoparticle. Environmental Science \& Technology Letters, 2014, 1(3): 209-213.

[53] WU D, PENG S, ZHANG Y, et al. Enhanced As(III) sequestration using sulfide-modified nano-scale zero-valent iron with a characteristic core-shell structure: sulfidation and as distribution. ACS Sustainable Chemistry \& Engineering, 2018, 6(3): 3039-3048.

[54] MEFFRE A, RESPAUD M, CHAUDRET B, et al. Use of long chain amine as a reducing agent for the synthesis of high quality monodisperse iron(0) nanoparticles. Journal of Materials Chemistry, 2011, 21(35): 13464-13469.
[55] EGEBERG A, BLOCK T, FELDMANN C. Lithiumpyridinyldriven synthesis of high-purity zero-valent iron nanoparticles and their use in follow-up reactions. Small, 2019, 15(37): 1902321.

[56] LUO W, LIU H K, YANG J P, et al. Germanium nanograin decoration on carbon shell: boosting lithium-storage properties of silicon nanoparticles. Advanced Functional Materials, 2016, 26(43): 7800-7806.

[57] SUN Z, YANG J P, ZHAO D Y, et al. A versatile designed synthesis of magnetically separable nano-catalysts with well-defined core-shell nanostructures. Journal of Materials Chemistry A, 2014, 2(17): 6071-6074.

[58] WANG Q Q, JIANG W, YANG J P, et al. Iron nanoparticles in capsules: derived from mesoporous silica-protected Prussian blue microcubes for efficient selenium removal. Chemical Communications, 2018, 54(46): 5887-5890.

[59] JIAO J, WANG H, CAO L, et al. In situ confined growth based on a self-templating reduction strategy of highly dispersed $\mathrm{Ni}$ nanoparticles in hierarchical yolk-shell $\mathrm{Fe} @ \mathrm{SiO}_{2}$ structures as efficient catalysts. Chemistry-An Asian Journal, 2016, 11(24): 3534-3540.

[60] LAN Y, CHEN J L, YANG J P, et al. $\mathrm{Fe} / \mathrm{Fe}_{3} \mathrm{C}$ nanoparticledecorated $\mathrm{N}$-doped carbon nanofibers for improving the nitrogen selectivity of electrocatalytic nitrate reduction. Journal of Materials Chemistry A, 2020, 8(31): 15853-15863.

[61] LIANG H W, WEI W, FENG X, et al. Mesoporous metal-nitrogendoped carbon electrocatalysts for highly efficient oxygen reduction reaction. Journal of the American Chemical Society, 2013, 135(43): $16002-16005$

[62] XIAO M, ZHU J, XING W, et al. Meso/macroporous nitrogendoped carbon architectures with iron carbide encapsulated in graphitic layers as an efficient and robust catalyst for the oxygen reduction reaction in both acidic and alkaline solutions. Advanced Materials, 2015, 27(15): 2521-2527.

[63] WU Z Y, XU X X, YU S H, et al. Iron carbide nanoparticles encapsulated in mesoporous $\mathrm{Fe}-\mathrm{N}$-doped carbon nanofibers for efficient electrocatalysis. Angewandte Chemie-International Edition, 2015, 54(28): 8179-8183.

[64] LI Z, LI G, LI F, et al. Ionic liquids as precursors for efficient mesoporous iron-nitrogen-doped oxygen reduction electrocatalysts. Angewandte Chemie-International Edition, 2015, 54(5): 1494-1498.

[65] TENG W, FAN J W, ZHANG W X, et al. Nanoscale zero-valent iron in mesoporous carbon (nZVI@C): stable nanoparticles for metal extraction and catalysis. Journal of Materials Chemistry A, 2017, 5(9): 4478-4485.

[66] LI J, CHEN C, WANG X, et al. Nanoscale zero-valent iron particles modified on reduced graphene oxides using a plasma technique for Cd(II) removal. Journal of the Taiwan Institute of Chemical Engineers, 2016, 59: 389-394.

[67] WANG C, LUO H, CHEN S, et al. Removal of As(III) and As(V) from aqueous solutions using nanoscale zero valent iron-reduced graphite oxide modified composites. Journal of Hazardous Materials, 2014, 268: 124-131.

[68] KANEL S R, MANNING B, CHOI H, et al. Removal of arsenic(III) from groundwater by nanoscale zero-valent iron. Environmental Science \& Technology, 2005, 39(5): 1291-1298.

[69] TANG C, LING L, ZHANG W X. Pb(II) deposition-reductiongrowth onto iron nanoparticles induced by graphitic carbon nitride. Chemical Engineering Journal, 2020, 387: 124088.

[70] CHEN M, WANG H, YANG J P, et al. Achieving high-performance nitrate electrocatalysis with $\mathrm{PdCu}$ nanoparticles confined in nitrogendoped carbon coralline. Nanoscale, 2018, 10(40): 19023-19030.

[71] DUAN W, LI G, FENG C, et al. Highly active and durable carbon electrocatalyst for nitrate reduction reaction. Water Research, 2019, 161: 126-135.

[72] WANG J, LING L, ZHANG W X, et al. Nitrogen-doped iron for selective catalytic reduction of nitrate to dinitrogen. Science Bulletin, 2020, 65(11): 926-933. 